\title{
Experimental measurements of the detonation wave profile in a TATB based explosive
}

\author{
V. Bouyer, M. Doucet, and L. Decaris \\ CEA, DAM, 37260 Le Ripault, France
}

\begin{abstract}
We report results of the experimental measurements of the detonation wave profile of the TATB based plastic bonded explosive T2 (97 w. \% of TATB) using VISAR and Heterodyne Velocimetry (HV - same as Photon Doppler Velocimetry). The experiment consists in initiating a detonation wave in a $15 \mathrm{~mm}$ diameter cylinder of explosive using an explosive wire detonator and an explosive booster. In order to obtain the particle velocity history in the reaction zone, we measure particle velocity at the interaction of the detonation front with an aluminized window or the free surface velocity of a metallic foil. Lithium Fluoride (LIF), PMMA and steel have been tested. Several shots have been performed for different lengths of explosive. We compare the VISAR and HV measurements. With LIF and steel, VISAR and HV diagnostics give very similar profiles. The ZND profile obtained on LIF is resolved with both techniques. With PMMA, HV gives a more accurate profile than VISAR in the reaction zone. There is no evidence of the influence of the explosive cylinder length.
\end{abstract}

\section{Introduction}

The purpose of this study is to improve the knowledge of the reaction zone of high explosives using an experimental approach. The short temporal duration of the Von Neumann spike and the early part of the reaction zone make these measurements difficult. We have already carried out measurements on nitromethane [1] using two laser interferometry techniques, VISAR and Heterodyne Velocimetry or HV (same as Photon Doppler Velocimetry). One of the main observations is that the measured peaks are always 10 to $20 \%$ below the estimated VN spike in nitromethane in spite of the high time resolution of the diagnostics (about 1 or $2 \mathrm{~ns}$ ).

The work described here deals with the experimental study of the detonation wave profile of the TATB plastic bonded based explosive T2 (97 w. \% of TATB) using also VISAR and HV. Fabry Perot interferometer, ORVIS and PDV measurements of the interaction of the detonation wave of PBX9502 with windows or foils have been previously accomplished respectively by Seitz et al. [2], Sheffield et al. [3] and Gustavsen et al. [4].

Our objective is to measure the particle velocity histories at the T2/window interface and to analyze the ability of VISAR and HV to give accurate profiles in the reaction zone. Several shots have been performed on steady 2D divergent flow configurations, enabling to study the interaction of the detonation front with a transparent window or a thin metal plate.

\section{Experimental set up}

The experiment consists in initiating a detonation wave in a cylinder of explosive using an explosive wire detonator and an explosive booster. There is no overdriven detonation in T2. In order to obtain

This is an Open Access article distributed under the terms of the Creative Commons Attribution-Noncommercial License 3.0, which permits unrestricted use, distribution, and reproduction in any noncommercial medium, provided the original work is properly cited. 


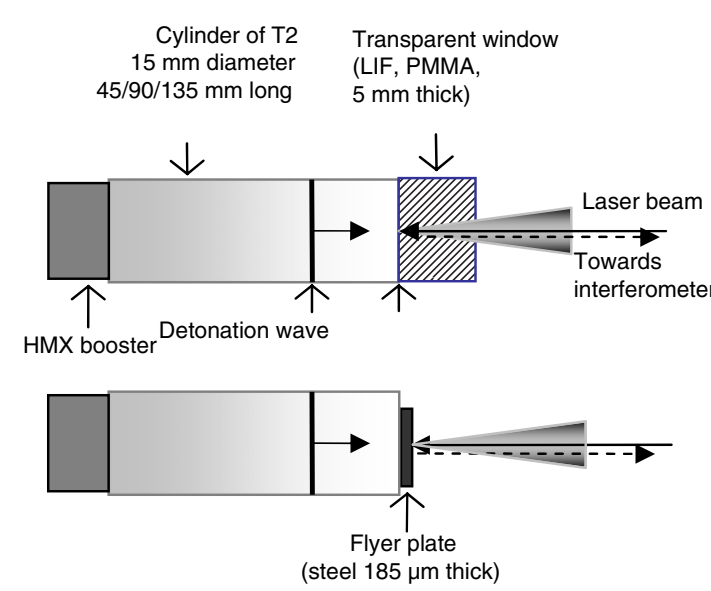

Fig. 1. Setup for the particle velocity measurements.

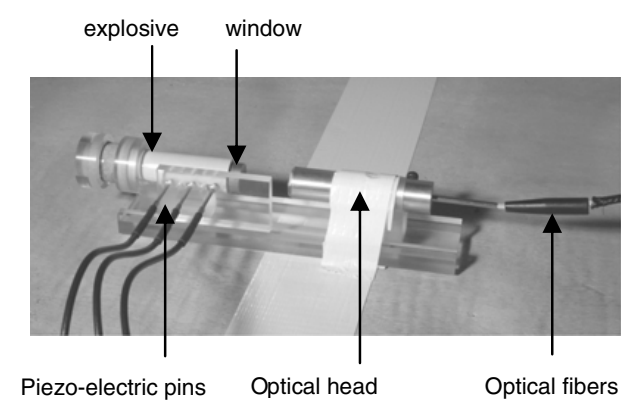

Fig. 2. Experimental device.

the particle velocity history in the reaction zone, we measure the interaction of the detonation front with a transparent window or a metallic foil (see Fig. 1 and Fig. 2).

The explosive/window interface is coated with aluminum to enable the reflection of the laser beam for the interferometry measurement. The thickness of the coating is less than $1 \mu \mathrm{m}$. The particle velocity profile of the explosive/window interface and of the foil free surface is measured along the detonation propagation axis.

The TATB cylinders have a $15 \mathrm{~mm}$ diameter and 45, 90 or $135 \mathrm{~mm}$ lengths. The HMX booster has a $18 \mathrm{~mm}$ diameter and $18 \mathrm{~mm}$ length.

Lithium Fluoride (LIF), PMMA, and steel are the tested "window materials". LIF and steel are a strong overmatch into T2 whereas PMMA is a strong undermatch. Therefore, in the event we would be able to determine the reaction zone length, the particle velocity profile at the explosive/LIF - Steel interface would give an underestimate of the length whereas the measurement on PMMA would give an overestimate.

The particle velocity history measurement is performed using VISAR and Heterodyne Veloci metry. VALYN VISAR system has a time resolution of about 1 or $2 \mathrm{~ns}$ and the accuracy is about $2 \%$ on velocity [5]. HV is the CEA 2 lasers PDV system [6] at $1550 \mathrm{~nm}$. The acquisition bandwidth is $12 \mathrm{GHz}$ and signals are recorded with $20 \mathrm{ps}$ /point. The analysis is performed using a sliding window Fourier transform. The time resolution and the velocity accuracy obtained after analysis of the experiments described here are respectively $1 \mathrm{~ns}$ and $\pm 50 \mathrm{~m} / \mathrm{s}$. When measuring the interface velocity through the LIF window, a correction is applied both for VISAR and HV. Corrections factors at $532 \mathrm{~nm}$ and $1550 \mathrm{~nm}$ are given by Jensen et al. [7,8].

For all shots except shot 8310 , the collection of the signal is performed using a $20 \mathrm{~mm}$ focal length lens and a magnification of 1 . VISAR fibers have a $200 \mu \mathrm{m}$ core diameter and HV fibers have a $9 \mu \mathrm{m}$ core diameter. For shot 8310, the collection is done with an OZ optics probe (LPC-01-1550-9/125-S0.44-2AS-60-3A-1-3).

\section{Results and analysis}

Results of the experiments are presented for the two window materials and for the steel foil. HV and VISAR signals are compared.

Direct numerical simulations have been also performed, using a wide range EOS/reaction rate model calibrated to reproduce both the strong shock initiation and the extended celerity-curvature law [9]. 
Table 1. Summary of the shots.

\begin{tabular}{|c|c|c|c|}
\hline Shot & Window material & Explosive Length (mm) & Diagnostics \\
\hline 8409 & LIF & 90 & VISAR/HV \\
\hline 8609 & LIF & 90 & HV \\
\hline 1310 & LIF & 45 & VISAR/ HV \\
\hline 1410 & PMMA & 45 & VISAR/ HV \\
\hline 1510 & Steel & 45 & VISAR/ HV \\
\hline 8210 & LIF & 135 & HV \\
\hline 8310 & PMMA & 45 & HV \\
\hline
\end{tabular}

Shots numbers, window materials, lengths of the T2 cylinder and laser velocimetry techniques are given in Table 1.

\subsection{T2/LIF window}

Figure 3 shows the particle velocity profiles obtained at T2/LIF interface for shots 8409, 8609, 1310 and 8210 . For all shots, VISAR and HV give very similar profiles and the ZND profile is resolved with both techniques. The amplitude of the Von Neumann (VN) spike corresponds to the theoretical value which is about $2200 \mathrm{~m} / \mathrm{s}$ at the LIF interface.

The identical shape of the profiles in the release part obtained for all the configurations gives no evidence of the influence of the explosive cylinder length.

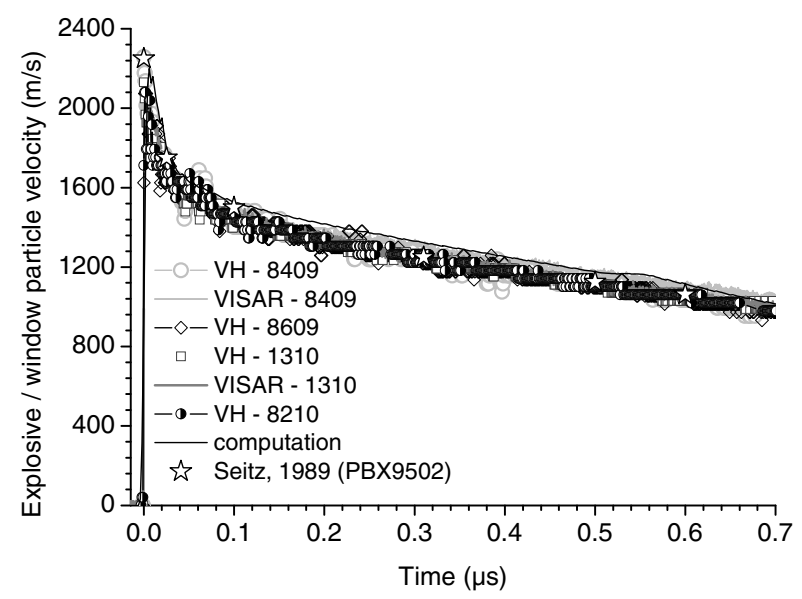

Fig. 3. Particle velocity profiles at T2/LIF interface obtained with VISAR and HV.

Our measurements on T2 can be compared to those obtained by Seitz et al. in 1989 [1]. Seitz measured the interaction of the detonation wave propagating in a $14 \mathrm{~mm}$ diameter cylinder of PBX9502 with a LIF window. The particle velocity histories show that the two plastic bonded TATB based explosive behave similarly.

\subsection{T2/PMMA window}

In the case of the interaction of the T2 detonation wave with a PMMA window, two shots have been performed (shot 1410 and 8310). HV gives more accurate profiles than VISAR in the reaction zone (see Fig. 4). VISAR does not record the VN spike. On the contrary, HV signal gives a VN spike that corresponds to the theoretical value which is about $2900-3000 \mathrm{~m} / \mathrm{s}$ at the PMMA interface. 


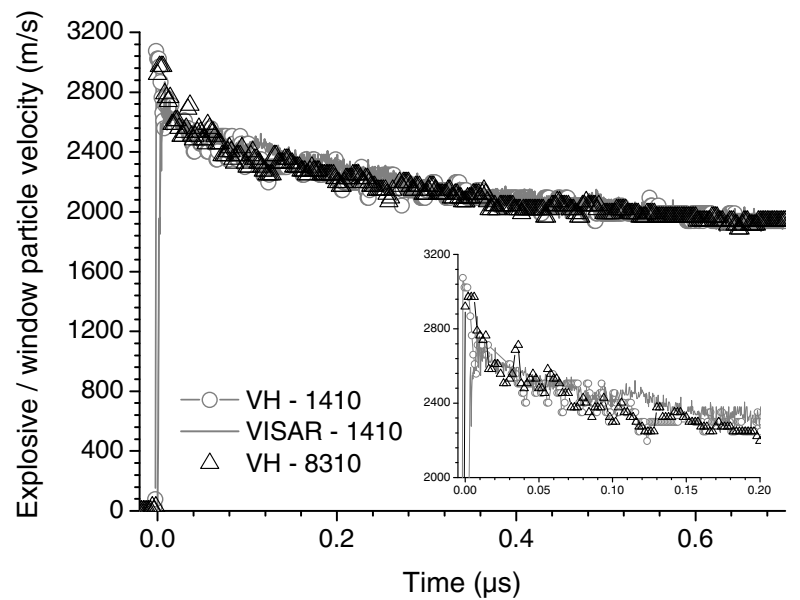

Fig. 4. Particle velocity profiles at T2/PMMA interface obtained with VISAR and HV.

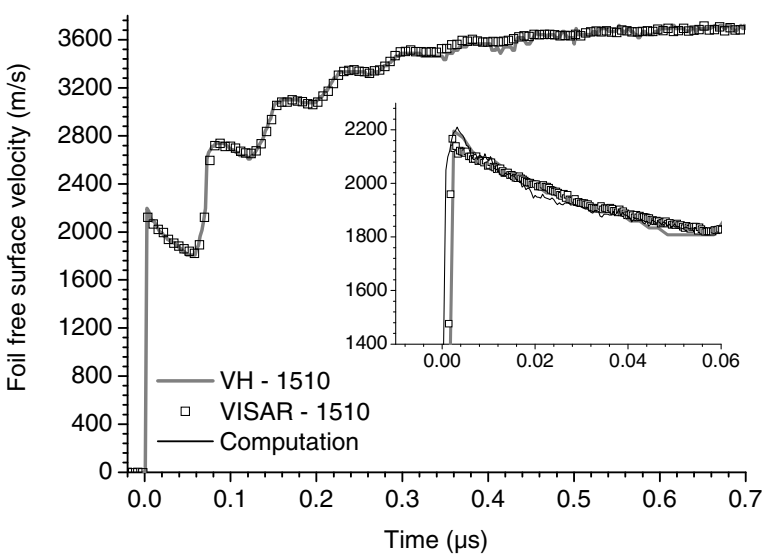

Fig. 5. Steel free surface velocity obtained with VISAR and VH.

Velocity profiles are more disturbed than those obtained with LIF. No explanation of this phenomenon is given for the moment.

\subsection{T2/steel foil}

The steel foil free surface velocity profiles obtained with HV and VISAR are represented on Fig. 5.

The two signals match very well although some discrepancies can be observed between 0.3 and $0.6 \mu$ s. Compared to VH, VISAR gives an average signal. That can be explained by the size of the measurement spot. Indeed, the optical probe is the same for both measurements and the magnification is equal to 1 . Therefore, the size of the measurement spot corresponds to the fiber core diameter, which is $200 \mu \mathrm{m}$ for VISAR vs. $9 \mu \mathrm{m}$ for HV.

For the three configurations (LIF, PMMA and steel), the computations of the particle velocity profiles have been compared with the experimental signal. Profiles only fit for LIF and steel. We can consider that EOS of both materials are relevant. In the case of PMMA, computation profiles do not fit the measured signal, probably because the EOS of PMMA that was used in the calculation does not correspond to the real window material. 


\section{Discussion and conclusions}

We have performed several experiments in order to study the particle velocity profile of the detonation front of the plastic bonded TATB based explosive T2. Particle velocity histories of the interface between explosive and LIF or PMMA transparent windows and of a steel foil free surface have been obtained using VISAR and Heterodyne Velocimetry. Steady two-dimensional cylinder configurations of various lengths have been tested.

Several observations can be made.

In the reaction zone, profiles are characteristic from a sharp shock, followed by a fast decrease then a slow decrease of the particle velocity, which supports the idea that there is fast and slow chemistry in the reaction zone, as in nitromethane (NM) [1]. Von Neumann spike amplitudes are well resolved when using LIF as the window material and are of the right order of magnitude, unlike what was obtained with NM.

In the range of lengths tested, there is no evidence of the influence of the length of the explosive on the particle velocity profile. Therefore, in contrary to Gustavsen results of plate impact experiments on plastic bonded explosives [4], the estimation of the reaction zone length using the change of slope in the release wave part of the profile is not relevant here.

As what was observed on NM [1], these experiments on TATB show that VISAR and VH techniques give very similar profiles. The main difference with the previous work on NM is that in more cases, both techniques are able to record VN spike. With the actual retrieving software, HV provides the same time resolution as VISAR but the experiments performed on T2 and PMMA display VH could be somehow more accurate than VISAR in the ZND region.

Future work on this subject will consist in reducing the lengths of the T2 explosive samples in order to enhance the effect of the release wave on the particle velocity profiles, and to try to estimate the reaction zone length which is about $1-2 \mathrm{~mm}$ for plastic bonded TATB based explosives.

As $\mathrm{HV}$ is a recent technique, the analysis of the measurements can certainly be improved. We intend to test new retrieving methods of the Heterodyne Velocimetry signals that are developed at CEA, DAM, DIF.

\section{References}

1. V. Bouyer, S.A. Sheffield, D.M. Dattelbaum, R.L. Gustaven, D.B. Stahl, M. Doucet, L. Decaris, Proceedings of Shock Compression and Condensed Matter -2009, pp. 177-180, 2009.

2. W.L. Seitz, H.L. Stacy, R. Engelke, P.K. Tang, J. Wackerle, Proceedings of the $9^{\text {th }}$ International Detonation Symposium, 1989.

3. S.A. Sheffield, D.D. Bloomquist, C.M. Tarver, J. Chem. Physics, 80 (8), 3831-3844, 1984.

4. R.L. Gustavsen, B.D. Bartram, N.J. Sanchez, Proceedings of Shock Compression and Condensed Matter-2009, pp. 253-256, 2009.

5. L.M. Barker, R.E. Hollenbach, J. Appl. Phys., 43 (11), 4669-4675 (1972).

6. P. Mercier, J. Bénier, P.A. Frugier, G. Contencin, J. Veaux, S. Lauriot-Basseuil, Proceedings SPIE, 7126 (2008).

7. B.J. Jensen, D.B. Holtkamp, P.A. Rigg, J. Appl. Phys., 101, 013523 (2007).

8. B.J. Jensen, D.B. Holtkamp, P.A. Rigg, D.H. Dolan, J. Appl. Phys., 106, 049901 (2009).

9. C. Matignon, R. Sorin, O. Bozier, Proceedings of the $14^{\text {th }}$ International Detonation Symposium, 2010. 